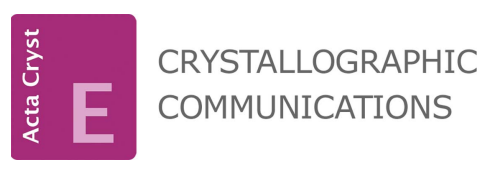

ISSN 2056-9890

Received 8 August 2018

Accepted 28 August 2018

Edited by J. Ellena, Universidade de Sâo Paulo, Brazil

Keywords: crystal structure; copper(II); hydroxamates; pivalate derivatives; mononuclear complexes.

CCDC reference: 1864482

Supporting information: this article has supporting information at journals.iucr.org/e

\section{Crystal structure of bis(pivaloylhydroxamato- $\left.\kappa^{2} O, O^{\prime}\right) \operatorname{copper}(\mathrm{II})$}

\author{
Kateryna Goleva, ${ }^{a}$ Dina Naumova, ${ }^{a}$ Anna Pavlishchuk, ${ }^{a *}$ Anthony W. Addison ${ }^{\mathrm{b}}$ and \\ Matthias Zeller ${ }^{\mathrm{c}}$

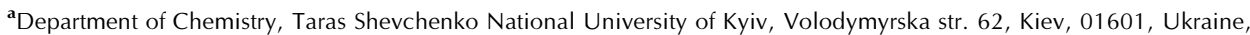 \\ b Department of Chemistry, Drexel University, Philadelphia, PA 19104-2816, USA, and ' Department of Chemistry, \\ Purdue University, 560 Oval Dr., West Lafayette, IN 47907-2084, USA. *Correspondence e-mail: annpavlis@ukr.net
}

Reaction of copper(II) nitrate with pivaloylhydroxamic acid yielded the title compound, $\left[\mathrm{Cu}(\text { pivHA })_{2}\right.$ ] (where pivHA ${ }^{-}$is pivaloyl hydroxamate, $\mathrm{C}_{5} \mathrm{H}_{10} \mathrm{NO}_{2}$ ). The centrosymmetric mononuclear complex consists of a $\mathrm{Cu}^{\mathrm{II}}$ ion, which is located on a center of inversion, with two coordinated pivaloyl hydroxamate monoanions. The $\mathrm{Cu}^{\mathrm{II}}$ ion has a square-planar coordination environment consisting of four $\mathrm{O}$ atoms - two carbonyl $\mathrm{O}$ atoms and two hydroxamate $\mathrm{O}$ atoms from two hydroxamate pivHA ${ }^{-}$ligands. The pivHA $^{-}$anions are coordinated to copper(II) in a trans-mode, forming two five-membered $O, O^{\prime}$ chelate rings.

\section{Chemical context}

Numerous studies over the past decade of various hydroxamate complexes with $3 d$ and $4 f$ metal ions have been inspired by their potential applications in molecular magnetism, luminescence, adsorption and catalysis (Ostrowska et al., 2016; Pavlishchuk et al., 2015). The ability of further functionalized hydroxamic acids to serve as bridging ligands and to form polynuclear species with different structural motifs has been comprehensively examined in recent years (Mezei et al., 2007; Pavlishchuk et al., 2018; Odarich et al., 2016; McDonald et al., 2014, 2015; Gaynor et al., 2002). Studies of simple unsubstituted hydroxamic acids have been undertaken because of their possible application as mimics of mononuclear iron(III) siderophores (Marmion et al., 2004). As a result of the potentially multiple coordination modes of unsubstituted hydroxamic acids, they can also lead to the formation of polynuclear assemblies (Tirfoin et al., 2014). However, reactions of unsubstituted hydroxamic acids with transition metal ions lead mainly to the formation of octahedral 1:3 (Abu-Dari et al., 1979) or square-planar 1:2 (Baughman et al., 2000) complexes with the hydroxamate in an $O, O^{\prime}$-coordination mode. The ability of pivalic acid itself to form polynuclear metallamacrocyclic complexes with various metal ions is well known (Vitórica-Yrezábal et al., 2017; Garlatti et al., 2018). The aim of the current work was to investigate if a tert-butyl-substituted hydroxamic acid (i.e. the hydroxamate analogue of pivalic acid) could be used as a scaffold for the preparation of polynuclear copper(II) complexes. 


\section{Structural commentary}

Crystals of the title compound $\mathbf{1}$ were obtained by reaction of copper(II) nitrate hexahydrate with pivaloylhydroxamic acid in methanol.

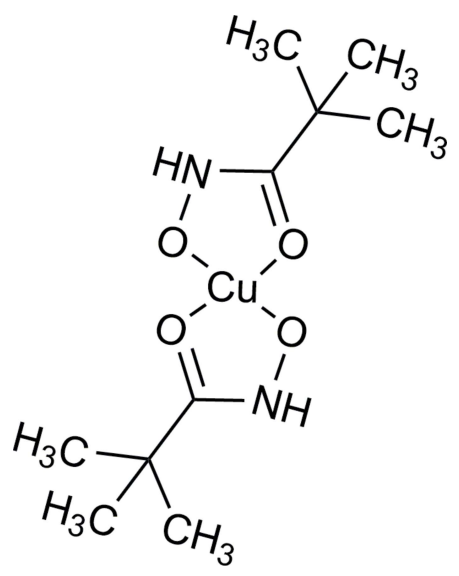

Complex 1 crystallizes in the space group $I 4_{1} / a$, with eight $\left[\mathrm{Cu}(\text { pivHA })_{2}\right]$ complex molecules per unit cell. The $\left[\mathrm{Cu}(\text { pivHA })_{2}\right]$ molecules are centrosymmetric, with the copper ion located on an inversion center. Each $\left[\mathrm{Cu}(\text { pivHA })_{2}\right]$ molecule contains one copper(II) ion in a square-planar coordination environment generated by the coordination of two pivaloylhydroxamate monoanions, forming five-membered chelate rings through both the carbonyl and hydroxamate $\mathrm{O}$ atoms (Fig. 1). The centrosymmetric nature of the complex forces the copper(II) ions to be exactly coplanar with the four donor $\mathrm{O}$ atoms, $\mathrm{O} 1 \mathrm{O} 2 \mathrm{O} 1^{\mathrm{i}} \mathrm{O} 2^{\mathrm{i}}$ [symmetry code: (i) $-x, 1-y$, $-z]$, and the two pivHA ${ }^{-}$monoanions in $\left[\mathrm{Cu}(\text { pivHA })_{2}\right]$ are necessarily mutually trans-coordinated. The axial positions of the copper(II) ions remain unoccupied. The $\mathrm{Cu}-\mathrm{O}_{\text {carbonyl }}$ and $\mathrm{Cu}-\mathrm{O}_{\text {hydroxamate }}$ bond lengths are typical for copper(II) hydroxamate or oximate complexes (Buvailo et al., 2012; Pavlishchuk et al., 2017a,b) (Table 1). The hydroxamate $\mathrm{N}-\mathrm{H}$ groups remain protonated and are not involved in metal coordination. Deprotonation of the $\mathrm{N}-\mathrm{H}$ groups could

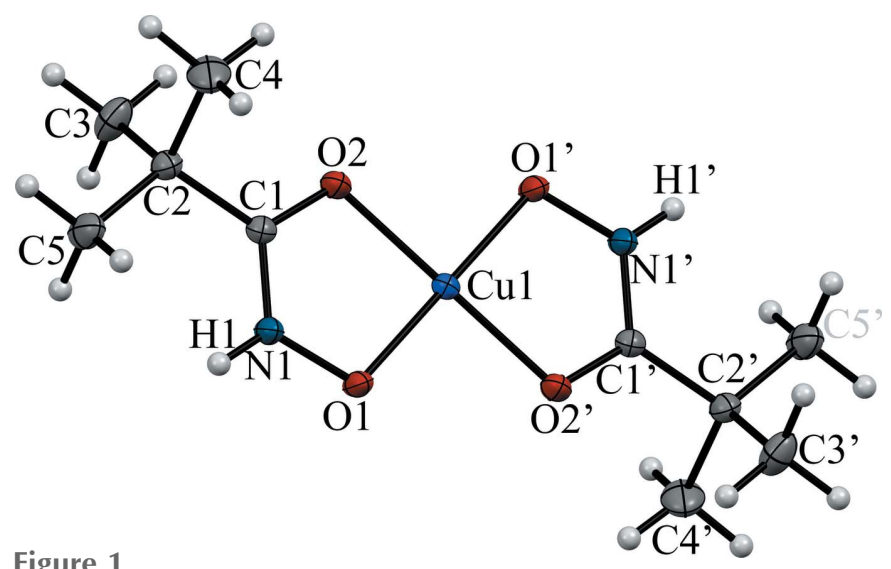

Figure 1

The molecular structure of complex $\mathbf{1}$ showing the neutral centrosymmetric fragment $\left[\mathrm{Cu}(\mathrm{pivHA})_{2}\right]$, along with the atom labelling. Displacement ellipsoids are at the $50 \%$ probability level. Symmetry code: (') $-x$, $1-y,-z$.
Table 1

Selected geometric parameters $\left(\AA,{ }^{\circ}\right)$.

\begin{tabular}{lclc}
\hline $\mathrm{C} 1-\mathrm{O} 2$ & $1.2821(13)$ & $\mathrm{O} 1-\mathrm{Cu} 1$ & $1.8899(8)$ \\
$\mathrm{C} 1-\mathrm{N} 1$ & $1.3066(14)$ & $\mathrm{O} 2-\mathrm{Cu} 1$ & $1.9244(8)$ \\
$\mathrm{N} 1-\mathrm{O} 1$ & $1.3764(12)$ & & \\
& & & \\
$\mathrm{O} 1-\mathrm{Cu} 1-\mathrm{O} 1^{\mathrm{i}}$ & $180(5)$ & $\mathrm{O} 1-\mathrm{Cu} 1-\mathrm{O}^{\mathrm{i}}$ & $95.16(3)$ \\
$\mathrm{O} 1-\mathrm{Cu} 1-\mathrm{O} 2$ & $84.84(3)$ & $\mathrm{O} 1^{\mathrm{i}}-\mathrm{Cu} 1-\mathrm{O} 2^{\mathrm{i}}$ & $84.84(3)$ \\
$\mathrm{O} 1^{\mathrm{i}}-\mathrm{Cu} 1-\mathrm{O} 2$ & $95.16(3)$ & $\mathrm{O} 2-\mathrm{Cu} 1-\mathrm{O} 2^{\mathrm{i}}$ & 180 \\
\hline
\end{tabular}

Symmetry code: (i) $-x,-y+1,-z$.

Table 2

Hydrogen-bond geometry $\left(\AA{ }^{\circ}\right)$.

\begin{tabular}{lllll}
\hline$D-\mathrm{H} \cdots A$ & $D-\mathrm{H}$ & $\mathrm{H} \cdots A$ & $D \cdots A$ & $D-\mathrm{H} \cdots A$ \\
\hline $\mathrm{N} 1-\mathrm{H} 1 \cdots \mathrm{O} 1^{\mathrm{ii}}$ & 0.88 & 1.90 & $2.7185(13)$ & 154 \\
\hline
\end{tabular}

Symmetry code: (ii) $y-\frac{1}{4},-x+\frac{1}{4},-z+\frac{1}{4}$.

possibly be achieved at higher $\mathrm{pH}$ without hydrolysis of hydroxamic acid, which might aid in the formation of polynuclear complexes.

\section{Supramolecular features}

Adjacent $\left[\mathrm{Cu}(\text { pivHA })_{2}\right]$ complexes are connected to each other via $\mathrm{N} 1-\mathrm{H} 1 \cdots \mathrm{O} 1^{\mathrm{ii}}$ hydrogen bonds between the hydroxamate $\mathrm{N}-\mathrm{H}$ group of one complex molecule and a deprotonated hydroxamate oxygen of an adjacent $\left[\mathrm{Cu}(\text { pivHA })_{2}\right]$ molecule (Table 2, Fig. 2). Four of these $\mathrm{N}-\mathrm{H} \cdots \mathrm{O}$ hydrogen bonds connect molecules into tetramers arranged around a fourfold rotoinversion center. The $\mathrm{N}-\mathrm{H}$ group of the second hydroxamate ligand of each complex creates an equivalent tetramer trans across the copper ion, thus creating an infinite three-dimensional network of corner-connected tetramers (with the copper ions acting as the bridging element, Fig. 3).

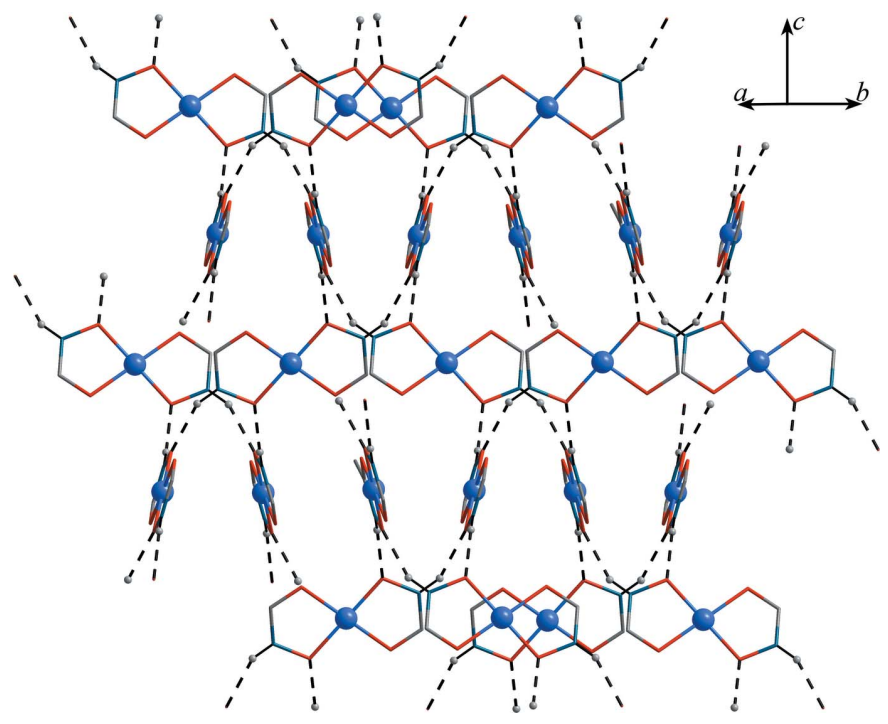

Figure 2

A fragment of the lattice of complex $\mathbf{1}$, showing the intramolecular hydrogen-bonding connections (dashed lines) between the $\left[\mathrm{Cu}(\text { pivHA })_{2}\right]$ molecules. The tert-butyl groups are omitted for clarity. 


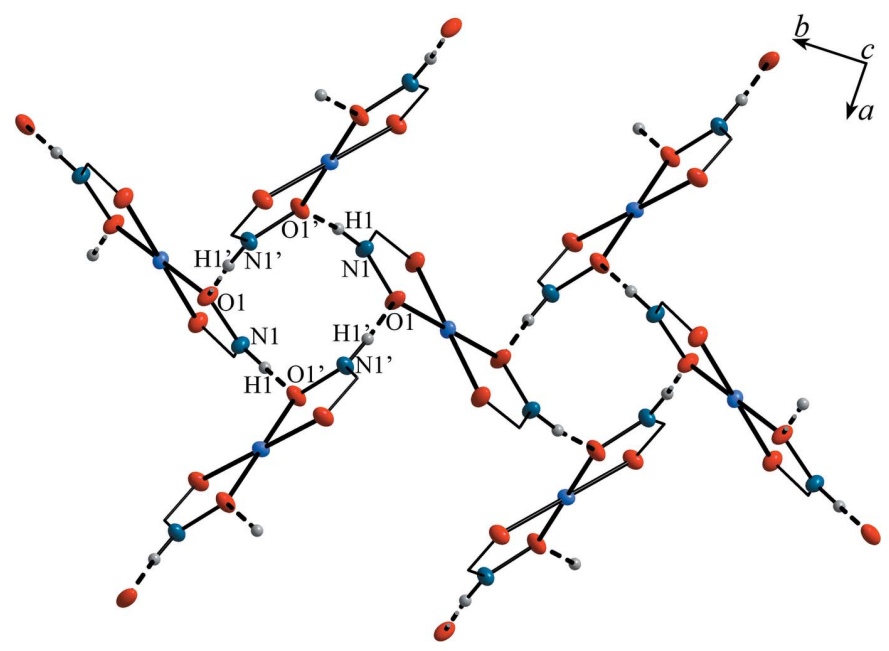

Figure 3

A fragment of the packing of complex $\mathbf{1}$, showing the formation of supramolecular tetramers $\left[\mathrm{Cu}(\text { pivHA })_{2}\right]_{4}$ formed by hydrogen bonds. The tert-butyl groups are omitted for clarity.

\section{Database survey}

The Cambridge Structural Database (CSD, Version 5.27, updated in August 2012; Groom et al., 2016) contains one report with structural information for pivaloylhydroxamic acid (CCDC 1155138; Due et al., 1987). Though the survey did not contain any information about complexes with pivaloylhydroxamic acid, there are two reports devoted to structural studies of $\mathrm{Th}^{4+}$ (1180613 and 1180614; Smith \& Raymond, 1981) and $\mathrm{MoO}_{2}{ }^{2+}$ (763210-763214; Dzyuba et al., 2010) complexes with structurally similar ligands ( $N$-isopropyl-2,2dimethylpropanehydroxamate, $\quad N$-isopropyl-3,3-dimethylbutanehydroxamate and decano-, $N$-methyl-decano-, $N$ methyl-hexano-, $N$-methyl-1-adamantano- or $N$-tert-butylhexanohydroxamates, respectively). It should be mentioned that coordination of hydroxamate ligands in the $O, O^{\prime}$ chelating mode is quite typical (Tedeschi et al., 2003; Seitz et $a l ., 2007 a, b$; Brewer \& Sinn, 1981) and the CSD contains many records with such binding in various mononuclear bis-hydroxamate complexes (e.g. Drovetskaia et al., 1996; Li et al., 2004; Fisher et al., 1989; Harrison et al., 1976), which are usually coordinated in the trans- mode with respect to each other (Gaynor et al., 2001; Lasri et al., 2012; Casellato et al., 1984).

\section{Synthesis and crystallization}

A solution of pivaloylhydroxamic acid $(23.4 \mathrm{mg}, 0.20 \mathrm{mmol})$ in $5 \mathrm{~mL}$ of methanol was added to copper(II) nitrate hexahydrate $(29.6 \mathrm{mg}, 0.10 \mathrm{mmol})$ in $5 \mathrm{~mL}$ of methanol. The resulting blue solution was stirred for $30 \mathrm{~min}$. at room temperature, filtered and left for slow evaporation. After a week, blue crystals suitable for single crystal X-ray analysis had formed. Yield: $23 \mathrm{mg}$ (78\%). Elemental analysis C:H:N Expected (calculated): 40.75 (40.60): 7.03 (6.81): 9.22 (9.47). IR in $\mathrm{KBr}$ pellets $\left(\mathrm{cm}^{-1}\right): 3400\left(v_{\mathrm{N}-\mathrm{H}}\right) ; 3196-3040\left(v_{\mathrm{O}-\mathrm{H}}\right.$, likely due to the presence of $\mathrm{N} 1-\mathrm{H} 1 \cdots \mathrm{O} 1^{\mathrm{ii}}$ hydrogen bonds); 1595
Table 3

Experimental details.

\begin{tabular}{|c|c|}
\hline \multicolumn{2}{|l|}{ Crystal data } \\
\hline Chemical formula & {$\left[\mathrm{Cu}\left(\mathrm{C}_{5} \mathrm{H}_{10} \mathrm{NO}_{2}\right)_{2}\right]$} \\
\hline$M_{\mathrm{r}}$ & 295.82 \\
\hline Crystal system, space group & Tetragonal, $I 4_{1} / a$ \\
\hline Temperature $(\mathrm{K})$ & 100 \\
\hline$a, c(\AA)$ & $12.8059(5), 17.7051(8)$ \\
\hline$V\left(\AA^{3}\right)$ & $2903.5(3)$ \\
\hline$Z$ & 8 \\
\hline Radiation type & Mo $K \alpha$ \\
\hline$\mu\left(\mathrm{mm}^{-1}\right)$ & 1.51 \\
\hline Crystal size $(\mathrm{mm})$ & $0.35 \times 0.35 \times 0.29$ \\
\hline \multicolumn{2}{|l|}{ Data collection } \\
\hline Diffractometer & Bruker D8 Quest CMOS \\
\hline Absorption correction & $\begin{array}{l}\text { Multi-scan (SADABS; Krause et } \\
\text { al., 2015) }\end{array}$ \\
\hline$T_{\min }, T_{\max }$ & $0.656,0.747$ \\
\hline $\begin{array}{l}\text { No. of measured, independent and } \\
\text { observed }[I>2 \sigma(I)] \text { reflections }\end{array}$ & $24433,2764,2444$ \\
\hline$R_{\text {int }}$ & 0.035 \\
\hline$(\sin \theta / \lambda)_{\max }\left(\AA^{-1}\right)$ & 0.769 \\
\hline \multicolumn{2}{|l|}{ Refinement } \\
\hline$R\left[F^{2}>2 \sigma\left(F^{2}\right)\right], w R\left(F^{2}\right), S$ & $0.029,0.074,1.19$ \\
\hline No. of reflections & 2764 \\
\hline No. of parameters & 82 \\
\hline $\mathrm{H}$-atom treatment & $\mathrm{H}$-atom parameters constrained \\
\hline$\Delta \rho_{\max }, \Delta \rho_{\min }\left(\mathrm{e} \AA^{-3}\right)$ & $0.46,-0.48$ \\
\hline
\end{tabular}

Computer programs: APEX2 and, SAINT (Bruker, 2014), SHELXS97 (Sheldrick, 2008), SHELXL2018 (Sheldrick, 2015), shelXle (Hübschle et al., 2011), Mercury (Macrae et al., 2006) and publCIF (Westrip, 2010).

and $1503\left(v_{\text {amid I }}\right) ; 1330,1220$ and $1053\left(v_{\mathrm{C}-\mathrm{C}}\right.$ and $\left.v_{-\mathrm{C}-\mathrm{N}}\right) ; 963$ $\left(v_{\mathrm{N}-\mathrm{O}}\right)$.

\section{Refinement}

Crystal data, data collection and structure refinement details are summarized in Table 3. $\mathrm{H}$ atoms attached to carbon and nitrogen atoms were positioned geometrically and constrained to ride on their parent atoms: $\mathrm{C}-\mathrm{H}=0.98 \AA$ with $U_{\text {iso }}(\mathrm{H})=$ $1.5 U_{\text {eq }}(\mathrm{C})$ and $\mathrm{N}-\mathrm{H}=0.88 \AA$ with $U_{\text {iso }}(\mathrm{H})=1.2 U_{\text {eq }}(\mathrm{N})$. Methyl $\mathrm{H}$ atoms were allowed to rotate but not to tip to best fit the experimental electron density.

\section{Acknowledgements}

AWA thanks Drexel University for support.

\section{Funding information}

Funding for this research was provided by: National Science Foundation, Division of Materials Research (grant No. 1337296 to Matthias Zeller).

\section{References}

Abu-Dari, K., Ekstrand, J. D., Freyberg, D. P. \& Raymond, K. N. (1979). Inorg. Chem. 18, 108-112.

Baughman, R. G., Brink, D. J., Butler, J. M. \& New, P. R. (2000). Acta Cryst. C56, 528-531.

Brewer, G. A. \& Sinn, E. (1981). Inorg. Chem. 20, 1823-1830. 
Bruker (2014). APEX2 and SAINT. Bruker AXS Inc., Madison, Wisconsin, USA.

Buvailo, A. I., Pavlishchuk, A. V., Penkova, L. V., Kotova, N. V. \& Haukka, M. (2012). Acta Cryst. E68, m1480-m1481.

Casellato, U., Vigato, P. A., Tamburini, S., Graziani, R. \& Vidali, M. (1984). Inorg. Chim. Acta, 81, 47-54.

Drovetskaia, T. V., Yashina, N. S., Leonova, T. V., Petrosyan, V. S., Lorberth, J., Wocadlo, S., Massa, W. \& Pebler, J. (1996). J. Organomet. Chem. 507, 201-205.

Due, L., Rasmussen, H. \& Larsen, I. K. (1987). Acta Cryst. C43, 582585 .

Dzyuba, V. I., Koval, L. I., Bon, V. V. \& Pekhnyo, V. I. (2010). Polyhedron, 29, 2900-2906.

Fisher, D. C., Barclay-Peet, S. J., Balfe, C. A. \& Raymond, K. N. (1989). Inorg. Chem. 28, 4399-4406.

Garlatti, El. T., Guidi, A., Chiesa, S., Ansbro, S., Baker, J., Ollivier, H., Mutka, H., Timco, D. I., Vitorica-Yrezabal, E., Pavarini, P., Santini, G., Amoretti, G., Winpenny, S. \& Carretta, S. (2018). Chem. Sci. 9, 3555-3562.

Gaynor, D., Starikova, Z. A., Haase, W. \& Nolan, K. B. (2001). J. Chem. Soc. Dalton Trans. pp. 1578-1581.

Gaynor, D., Starikova, Z. A., Ostrovsky, S., Haase, W. \& Nolan, K. B. (2002). Chem. Commun. pp. 506-507.

Groom, C. R., Bruno, I. J., Lightfoot, M. P. \& Ward, S. C. (2016). Acta Cryst. B72, 171-179.

Harrison, P. G., King, T. J. \& Richards, J. A. (1976). J. Chem. Soc. Dalton Trans. pp. 1414-1418.

Hübschle, C. B., Sheldrick, G. M. \& Dittrich, B. (2011). J. Appl. Cryst. 44, 1281-1284.

Krause, L., Herbst-Irmer, R., Sheldrick, G. M. \& Stalke, D. (2015). J. Appl. Cryst. 48, 3-10.

Lasri, J., Gupta, S., da Silva, M. F. C. G. \& Pombeiro, A. J. L. (2012). Inorg. Chem. Commun. 18, 69-72.

Li, Q., Guedes da Silva, M. F. C. \& Pombeiro, A. J. L. (2004). Chem. Eur. J. 10, 1456-1462.

Macrae, C. F., Edgington, P. R., McCabe, P., Pidcock, E., Shields, G. P., Taylor, R., Towler, M. \& van de Streek, J. (2006). J. Appl. Cryst. 39, 453-457.

Marmion, C. J., Griffith, D. \& Nolan, K. B. (2004). Eur. J. Inorg. Chem. pp. 3003-3016.
McDonald, C., Sanz, S., Brechin, E. K., Singh, M. K., Rajaraman, G., Gaynor, D. \& Jones, L. F. (2014). RSC Adv. 4, 38182-38191.

McDonald, C., Williams, D. W., Comar, P., Coles, S. J., Keene, T. D., Pitak, M. B., Brechin, E. K. \& Jones, L. F. (2015). Dalton Trans. 44, 13359-13368.

Mezei, G., Zaleski, C. M. \& Pecoraro, V. L. (2007). Chem. Rev. 107, 4933-5003.

Odarich, I. A., Pavlishchuk, A. V., Kalibabchuk, V. A. \& Haukka, M. (2016). Acta Cryst. E72, 147-150.

Ostrowska, M., Fritsky, I. O., Gumienna-Kontecka, E. \& Pavlishchuk, A. V. (2016). Coord. Chem. Rev. 327-328, 304-332.

Pavlishchuk, A. V., Kolotilov, S. V., Zeller, M., Lofland, S. E. \& Addison, A. W. (2018). Eur. J. Inorg. Chem. doi: 1002ejic.201800461.

Pavlishchuk, A. V., Kolotilov, S. V., Zeller, M., Lofland, S. E., Kiskin, M. A., Efimov, N. N., Ugolkova, E. A., Minin, V. V., Novotortsev, V. M. \& Addison, A. W. (2017b). Eur. J. Inorg. Chem. pp. 48664878.

Pavlishchuk, A. V., Kolotilov, S. V., Zeller, M., Lofland, S. E., Thompson, L. K., Addison, A. W. \& Hunter, A. D. (2017a). Inorg. Chem. 56, 13152-13165.

Pavlishchuk, A. V., Satska, Y. A., Kolotilov, S. V. \& Fritsky, I. O. (2015). Curr. Inorg. Chem. 5, 5-25.

Seitz, M., Oliver, A. G. \& Raymond, K. N. (2007a). J. Am. Chem. Soc. 129, 11153-11160.

Seitz, M., Pluth, M. D. \& Raymond, K. N. (2007b). Inorg. Chem. 46, 351-353.

Sheldrick, G. M. (2008). Acta Cryst. A64, 112-122.

Sheldrick, G. M. (2015). Acta Cryst. C71, 3-8.

Smith, W. L. \& Raymond, K. N. (1981). J. Am. Chem. Soc. 103, 33413349.

Tedeschi, C., Azéma, J., Gornitzka, H., Tisnès, P. \& Picard, C. (2003). Dalton Trans. pp. 1738-1745.

Tirfoin, R., Chamoreau, L.-M., Li, Y., Fleury, B., Lisnard, L. \& Journaux, Y. (2014). Dalton Trans. 43, 16805-16817.

Vitórica-Yrezábal, I. J., Sava, D. F., Timco, G. A., Brown, M. S., Savage, M., Godfrey, H. G. W., Moreau, F., Schröder, M., Siperstein, F., Brammer, L., Yang, S., Attfield, M. P., McDouall, J. J. W. \& Winpenny, R. E. P. (2017). Angew. Chem. Int. Ed. 56, 5527-5530. Westrip, S. P. (2010). J. Appl. Cryst. 43, 920-925. 


\section{supporting information}

Acta Cryst. (2018). E74, 1384-1387 [https://doi.org/10.1107/S2056989018012227]

\section{Crystal structure of bis(pivaloylhydroxamato- $\left.\kappa^{2} O, O^{\prime}\right) \operatorname{copper(II)~}$}

\section{Kateryna Goleva, Dina Naumova, Anna Pavlishchuk, Anthony W. Addison and Matthias Zeller}

Computing details

Data collection: APEX2 (Bruker, 2014); cell refinement: SAINT (Bruker, 2014); data reduction: SAINT (Bruker, 2014); program(s) used to solve structure: SHELXS97 (Sheldrick, 2008); program(s) used to refine structure: SHELXL2018 (Sheldrick, 2015), shelXle (Hübschle et al., 2011); molecular graphics: Mercury (Macrae et al., 2006); software used to prepare material for publication: publCIF (Westrip, 2010).

Bis(pivaloylhydroxamato- $\left.\kappa^{2} O, O^{\prime}\right)$ copper(II)

Crystal data

$\left[\mathrm{Cu}\left(\mathrm{C}_{5} \mathrm{H}_{10} \mathrm{NO}_{2}\right)_{2}\right]$

$M_{r}=295.82$

Tetragonal, $I 4_{1} / a$

$a=12.8059(5) \AA$

$c=17.7051(8) \AA$

$V=2903.5(3) \AA^{3}$

$Z=8$

$F(000)=1240$

Data collection

Bruker AXS D8 Quest CMOS diffractometer

Radiation source: I-mu-S microsource X-ray tube

Laterally graded multilayer (Goebel) mirror monochromator

$\omega$ and phi scans

Absorption correction: multi-scan

(SADABS; Krause et al., 2015)

\section{Refinement}

Refinement on $F^{2}$

Least-squares matrix: full

$R\left[F^{2}>2 \sigma\left(F^{2}\right)\right]=0.029$

$w R\left(F^{2}\right)=0.074$

$S=1.19$

2764 reflections

82 parameters

0 restraints

Primary atom site location: structure-invariant direct methods
$D_{\mathrm{x}}=1.353 \mathrm{Mg} \mathrm{m}^{-3}$

Mo $K \alpha$ radiation, $\lambda=0.71073 \AA$

Cell parameters from 9939 reflections

$\theta=3.2-33.2^{\circ}$

$\mu=1.51 \mathrm{~mm}^{-1}$

$T=100 \mathrm{~K}$

Prism, blue

$0.35 \times 0.35 \times 0.29 \mathrm{~mm}$

$T_{\min }=0.656, T_{\max }=0.747$

24433 measured reflections

2764 independent reflections

2444 reflections with $I>2 \sigma(I)$

$R_{\text {int }}=0.035$

$\theta_{\max }=33.2^{\circ}, \theta_{\min }=3.2^{\circ}$

$h=-19 \rightarrow 19$

$k=-19 \rightarrow 19$

$l=-27 \rightarrow 27$

Secondary atom site location: difference Fourier map

Hydrogen site location: inferred from neighbouring sites

$\mathrm{H}$-atom parameters constrained

$w=1 /\left[\sigma^{2}\left(F_{\mathrm{o}}^{2}\right)+(0.0257 P)^{2}+3.2993 P\right]$

where $P=\left(F_{\mathrm{o}}^{2}+2 F_{\mathrm{c}}^{2}\right) / 3$

$(\Delta / \sigma)_{\max }<0.001$

$\Delta \rho_{\max }=0.46 \mathrm{e} \AA^{-3}$

$\Delta \rho_{\min }=-0.48 \mathrm{e} \AA^{-3}$ 


\section{Special details}

Geometry. All esds (except the esd in the dihedral angle between two 1.s. planes) are estimated using the full covariance matrix. The cell esds are taken into account individually in the estimation of esds in distances, angles and torsion angles; correlations between esds in cell parameters are only used when they are defined by crystal symmetry. An approximate (isotropic) treatment of cell esds is used for estimating esds involving l.s. planes.

Fractional atomic coordinates and isotropic or equivalent isotropic displacement parameters $\left(\AA^{2}\right)$

\begin{tabular}{lllll}
\hline & $x$ & $y$ & $z$ & $U_{\text {iso }} / U_{\text {eq }}$ \\
\hline C1 & $0.13160(8)$ & $0.34258(8)$ & $-0.02113(6)$ & $0.01465(18)$ \\
C2 & $0.20901(9)$ & $0.26772(9)$ & $-0.05643(7)$ & $0.01747(19)$ \\
C3 & $0.30109(12)$ & $0.33273(12)$ & $-0.08492(10)$ & $0.0327(3)$ \\
H3A & 0.349813 & 0.287558 & -0.112390 & $0.049^{*}$ \\
H3B & 0.337056 & 0.364691 & -0.041874 & $0.049^{*}$ \\
H3C & 0.275434 & 0.387597 & -0.118744 & $0.049^{*}$ \\
C4 & $0.15406(12)$ & $0.21475(12)$ & $-0.12341(8)$ & $0.0284(3)$ \\
H4A & 0.130218 & 0.268097 & -0.159159 & $0.043^{*}$ \\
H4B & 0.093923 & 0.174854 & -0.105007 & $0.043^{*}$ \\
H4C & 0.203005 & 0.167530 & -0.148793 & $0.043^{*}$ \\
C5 & $0.24745(12)$ & $0.18527(12)$ & $-0.00063(8)$ & $0.0274(3)$ \\
H5A & 0.187982 & 0.144200 & 0.017576 & $0.041^{*}$ \\
H5B & 0.281569 & 0.219691 & 0.042206 & $0.041^{*}$ \\
H5C & 0.297448 & 0.139024 & -0.025866 & $0.041^{*}$ \\
N1 & $0.10376(7)$ & $0.33335(8)$ & $0.04955(5)$ & $0.01550(17)$ \\
H1 & 0.128862 & 0.282995 & 0.078083 & $0.019^{*}$ \\
O1 & $0.03387(7)$ & $0.40497(7)$ & $0.07791(5)$ & $0.01837(16)$ \\
O2 & $0.09269(7)$ & $0.41676(7)$ & $-0.06079(5)$ & $0.01830(16)$ \\
Cu1 & 0.000000 & 0.500000 & 0.000000 & $0.01360(6)$ \\
& & & &
\end{tabular}

Atomic displacement parameters $\left(\AA^{2}\right)$

\begin{tabular}{lllllll}
\hline & $U^{11}$ & $U^{22}$ & $U^{33}$ & $U^{12}$ & $U^{13}$ & $U^{23}$ \\
\hline $\mathrm{C} 1$ & $0.0150(4)$ & $0.0146(4)$ & $0.0143(4)$ & $-0.0005(3)$ & $0.0017(3)$ & $0.0009(3)$ \\
$\mathrm{C} 2$ & $0.0180(5)$ & $0.0179(5)$ & $0.0166(5)$ & $0.0020(4)$ & $0.0038(4)$ & $0.0011(4)$ \\
$\mathrm{C} 3$ & $0.0242(6)$ & $0.0314(7)$ & $0.0424(8)$ & $-0.0013(5)$ & $0.0165(6)$ & $0.0028(6)$ \\
$\mathrm{C} 4$ & $0.0311(7)$ & $0.0303(6)$ & $0.0238(6)$ & $0.0075(5)$ & $-0.0015(5)$ & $-0.0098(5)$ \\
$\mathrm{C} 5$ & $0.0321(7)$ & $0.0275(6)$ & $0.0227(6)$ & $0.0144(5)$ & $0.0056(5)$ & $0.0046(5)$ \\
$\mathrm{N} 1$ & $0.0168(4)$ & $0.0156(4)$ & $0.0142(4)$ & $0.0034(3)$ & $0.0031(3)$ & $0.0026(3)$ \\
O1 & $0.0227(4)$ & $0.0186(4)$ & $0.0138(3)$ & $0.0077(3)$ & $0.0063(3)$ & $0.0033(3)$ \\
O2 & $0.0241(4)$ & $0.0172(4)$ & $0.0136(3)$ & $0.0045(3)$ & $0.0037(3)$ & $0.0035(3)$ \\
$\mathrm{Cu} 1$ & $0.01670(10)$ & $0.01244(9)$ & $0.01165(9)$ & $0.00085(6)$ & $0.00182(6)$ & $0.00171(6)$ \\
\hline
\end{tabular}

Geometric parameters $\left(\AA,{ }^{\circ}\right)$

\begin{tabular}{llll}
\hline $\mathrm{C} 1-\mathrm{O} 2$ & $1.2821(13)$ & $\mathrm{C} 4-\mathrm{H} 4 \mathrm{~B}$ & 0.9800 \\
$\mathrm{C} 1-\mathrm{N} 1$ & $1.3066(14)$ & $\mathrm{C} 4-\mathrm{H} 4 \mathrm{C}$ & 0.9800 \\
$\mathrm{C} 1-\mathrm{C} 2$ & $1.5141(16)$ & $\mathrm{C} 5-\mathrm{H} 5 \mathrm{~A}$ & 0.9800 \\
$\mathrm{C} 2-\mathrm{C} 5$ & $1.5275(18)$ & $\mathrm{C} 5-\mathrm{H} 5 \mathrm{~B}$ & 0.9800
\end{tabular}




\begin{tabular}{|c|c|c|c|}
\hline $\mathrm{C} 2-\mathrm{C} 3$ & $1.5290(18)$ & $\mathrm{C} 5-\mathrm{H} 5 \mathrm{C}$ & 0.9800 \\
\hline $\mathrm{C} 2-\mathrm{C} 4$ & $1.5368(18)$ & $\mathrm{N} 1-\mathrm{O} 1$ & $1.3764(12)$ \\
\hline $\mathrm{C} 3-\mathrm{H} 3 \mathrm{~A}$ & 0.9800 & $\mathrm{~N} 1-\mathrm{H} 1$ & 0.8800 \\
\hline $\mathrm{C} 3-\mathrm{H} 3 \mathrm{~B}$ & 0.9800 & $\mathrm{O} 1-\mathrm{Cu} 1$ & $1.8899(8)$ \\
\hline $\mathrm{C} 3-\mathrm{H} 3 \mathrm{C}$ & 0.9800 & $\mathrm{O} 2-\mathrm{Cu} 1$ & $1.9244(8)$ \\
\hline $\mathrm{C} 4-\mathrm{H} 4 \mathrm{~A}$ & 0.9800 & & \\
\hline $\mathrm{O} 2-\mathrm{C} 1-\mathrm{N} 1$ & $119.04(10)$ & $\mathrm{H} 4 \mathrm{~A}-\mathrm{C} 4-\mathrm{H} 4 \mathrm{C}$ & 109.5 \\
\hline $\mathrm{O} 2-\mathrm{C} 1-\mathrm{C} 2$ & $119.84(10)$ & $\mathrm{H} 4 \mathrm{~B}-\mathrm{C} 4-\mathrm{H} 4 \mathrm{C}$ & 109.5 \\
\hline $\mathrm{N} 1-\mathrm{C} 1-\mathrm{C} 2$ & $121.12(10)$ & $\mathrm{C} 2-\mathrm{C} 5-\mathrm{H} 5 \mathrm{~A}$ & 109.5 \\
\hline $\mathrm{C} 1-\mathrm{C} 2-\mathrm{C} 5$ & $112.43(10)$ & $\mathrm{C} 2-\mathrm{C} 5-\mathrm{H} 5 \mathrm{~B}$ & 109.5 \\
\hline $\mathrm{C} 1-\mathrm{C} 2-\mathrm{C} 3$ & $107.24(10)$ & $\mathrm{H} 5 \mathrm{~A}-\mathrm{C} 5-\mathrm{H} 5 \mathrm{~B}$ & 109.5 \\
\hline $\mathrm{C} 5-\mathrm{C} 2-\mathrm{C} 3$ & $109.95(12)$ & $\mathrm{C} 2-\mathrm{C} 5-\mathrm{H} 5 \mathrm{C}$ & 109.5 \\
\hline $\mathrm{C} 1-\mathrm{C} 2-\mathrm{C} 4$ & $107.35(10)$ & $\mathrm{H} 5 \mathrm{~A}-\mathrm{C} 5-\mathrm{H} 5 \mathrm{C}$ & 109.5 \\
\hline $\mathrm{C} 5-\mathrm{C} 2-\mathrm{C} 4$ & $109.97(11)$ & $\mathrm{H} 5 \mathrm{~B}-\mathrm{C} 5-\mathrm{H} 5 \mathrm{C}$ & 109.5 \\
\hline $\mathrm{C} 3-\mathrm{C} 2-\mathrm{C} 4$ & $109.81(11)$ & $\mathrm{C} 1-\mathrm{N} 1-\mathrm{O} 1$ & $117.82(9)$ \\
\hline $\mathrm{C} 2-\mathrm{C} 3-\mathrm{H} 3 \mathrm{~A}$ & 109.5 & $\mathrm{C} 1-\mathrm{N} 1-\mathrm{H} 1$ & 121.1 \\
\hline $\mathrm{C} 2-\mathrm{C} 3-\mathrm{H} 3 \mathrm{~B}$ & 109.5 & $\mathrm{O} 1-\mathrm{N} 1-\mathrm{H} 1$ & 121.1 \\
\hline $\mathrm{H} 3 \mathrm{~A}-\mathrm{C} 3-\mathrm{H} 3 \mathrm{~B}$ & 109.5 & $\mathrm{~N} 1-\mathrm{O} 1-\mathrm{Cu} 1$ & $108.18(6)$ \\
\hline $\mathrm{C} 2-\mathrm{C} 3-\mathrm{H} 3 \mathrm{C}$ & 109.5 & $\mathrm{C} 1-\mathrm{O} 2-\mathrm{Cu} 1$ & $110.11(7)$ \\
\hline $\mathrm{H} 3 \mathrm{~A}-\mathrm{C} 3-\mathrm{H} 3 \mathrm{C}$ & 109.5 & $\mathrm{O} 1-\mathrm{Cu} 1-\mathrm{O} 1^{\mathrm{i}}$ & $180.00(5)$ \\
\hline $\mathrm{H} 3 \mathrm{~B}-\mathrm{C} 3-\mathrm{H} 3 \mathrm{C}$ & 109.5 & $\mathrm{O} 1-\mathrm{Cu} 1-\mathrm{O} 2$ & $84.84(3)$ \\
\hline $\mathrm{C} 2-\mathrm{C} 4-\mathrm{H} 4 \mathrm{~A}$ & 109.5 & $\mathrm{O} 1-\mathrm{Cu} 1-\mathrm{O} 2$ & $95.16(3)$ \\
\hline $\mathrm{C} 2-\mathrm{C} 4-\mathrm{H} 4 \mathrm{~B}$ & 109.5 & $\mathrm{O} 1-\mathrm{Cu} 1-\mathrm{O} 2^{\mathrm{i}}$ & $95.16(3)$ \\
\hline $\mathrm{H} 4 \mathrm{~A}-\mathrm{C} 4-\mathrm{H} 4 \mathrm{~B}$ & 109.5 & 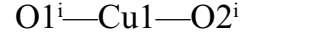 & $84.84(3)$ \\
\hline $\mathrm{C} 2-\mathrm{C} 4-\mathrm{H} 4 \mathrm{C}$ & 109.5 & $\mathrm{O} 2-\mathrm{Cu} 1-\mathrm{O} 2^{\mathrm{i}}$ & 180.0 \\
\hline $\mathrm{O} 2-\mathrm{C} 1-\mathrm{C} 2-\mathrm{C} 5$ & $179.56(11)$ & $\mathrm{C} 2-\mathrm{C} 1-\mathrm{N} 1-\mathrm{O} 1$ & $179.33(10)$ \\
\hline $\mathrm{N} 1-\mathrm{C} 1-\mathrm{C} 2-\mathrm{C} 5$ & $-0.14(16)$ & $\mathrm{C} 1-\mathrm{N} 1-\mathrm{O} 1-\mathrm{Cu} 1$ & $-0.49(12)$ \\
\hline $\mathrm{O} 2-\mathrm{C} 1-\mathrm{C} 2-\mathrm{C} 3$ & $58.59(15)$ & $\mathrm{N} 1-\mathrm{C} 1-\mathrm{O} 2-\mathrm{Cu} 1$ & $1.02(13)$ \\
\hline $\mathrm{N} 1-\mathrm{C} 1-\mathrm{C} 2-\mathrm{C} 3$ & $-121.11(13)$ & $\mathrm{C} 2-\mathrm{C} 1-\mathrm{O} 2-\mathrm{Cu} 1$ & $-178.69(8)$ \\
\hline $\mathrm{O} 2-\mathrm{C} 1-\mathrm{C} 2-\mathrm{C} 4$ & $-59.37(14)$ & $\mathrm{N} 1-\mathrm{O} 1-\mathrm{Cu} 1-\mathrm{O} 2$ & $0.79(7)$ \\
\hline $\mathrm{N} 1-\mathrm{C} 1-\mathrm{C} 2-\mathrm{C} 4$ & $120.94(12)$ & $\mathrm{N} 1-\mathrm{O} 1-\mathrm{Cu} 1-\mathrm{O} 2^{\mathrm{i}}$ & $-179.21(7)$ \\
\hline $\mathrm{O} 2-\mathrm{C} 1-\mathrm{N} 1-\mathrm{O} 1$ & $-0.37(16)$ & & \\
\hline
\end{tabular}

Symmetry code: (i) $-x,-y+1,-z$.

Hydrogen-bond geometry $\left(A,{ }^{\circ}\right)$

\begin{tabular}{lllll}
\hline$D-\mathrm{H}^{\cdots} \cdots A$ & $D-\mathrm{H}$ & $\mathrm{H} \cdots A$ & $D \cdots A$ & $D-\mathrm{H} \cdots A$ \\
\hline $\mathrm{N} 1-\mathrm{H} 1 \cdots \mathrm{O}^{\mathrm{ii}}$ & 0.88 & 1.90 & $2.7185(13)$ & 154 \\
\hline
\end{tabular}

Symmetry code: (ii) $y-1 / 4,-x+1 / 4,-z+1 / 4$. 